\title{
Growth, differentiation, transplantation and survival of human skeletal myofibers on biodegradable scaffolds
}

\author{
Lieven Thorrez ${ }^{\mathrm{a}, \mathrm{d}, *}$, Janet Shansky ${ }^{\mathrm{a}}$, Lin Wang ${ }^{\mathrm{a}}$, Loren Fast ${ }^{\mathrm{b}}$, Thierry VandenDriessche ${ }^{\mathrm{c}}$, \\ Marinee Chuah $^{\mathrm{c}}$, David Mooney ${ }^{\mathrm{d}}$, Herman Vandenburgh ${ }^{\mathrm{a}, \mathrm{d}}$ \\ ${ }^{a}$ Department of Pathology, Brown Medical School/The Miriam Hospital, RISE Building, 14 Third Street, Providence, RI 02906, USA \\ ${ }^{\mathrm{b}}$ Division of Hematology/Oncology, Brown Medical School/Rhode Island Hospital, 593 Eddy Street, Providence, RI 02903, USA \\ ${ }^{\mathrm{C}}$ Center for Transgene Technology and Gene Therapy, Flanders Institute for Biotechnology (VIB), Leuven University, Herestraat 49, 3000 Leuven, Belgium \\ ${ }^{\mathrm{d}}$ School of Engineering and Applied Sciences, Harvard University, Pierce Hall Room 325, 29 Oxford Street, Cambridge, MA 02138, USA
}

Received 16 May 2007; accepted 18 September 2007

\begin{abstract}
Skeletal muscle transplantation strategies for muscle repair or gene therapy involve either the injection of proliferating myoblasts followed by fusion with host myofibers or implantation of ex vivo differentiated myofibers; however, both implant procedures are associated with significant cell loss. Biodegradable porous, gas-foamed poly-lactide-co-glycolide (PLG) scaffolds have desirable characteristics for cell transfer and were used to study attachment, growth, differentiation and survival of human myogenic cells. Primary human myoblasts suspended in clinical grade extracellular matrixes (ECMs) and adhered to PLG scaffolds differentiated in vitro into high-density tropomyosin positive myofibers. An immunodeficient non-obese diabetic/severe combined immunodeficiency (NOD/SCID) mouse implant model was used to study the transfer and in vivo survival of differentiated human myofibers on these scaffolds. Scaffold rigidity allowed the myofibers to be maintained under tension in vitro and following subcutaneous transplantation in vivo. Following implantation, myofiber density on the PLG scaffolds decreased linearly by $78 \%$ over a 4-week period. ECM composed of either Tisseel ${ }^{\circledR}$ fibrin or Zyderm ${ }^{\circledR}$ collagen type I did not significantly affect in vivo cell viability over the 4-week period. Varying PLG scaffold microsphere content (10-100\%) also had little effect on cell survival in vivo. In contrast, when the residual NK cell population in the immunodeficient NOD/SCID mouse model was depleted with anti-asialo GM1 (ASGM1) antiserum, in vivo cell survival significantly increased from $22 \%$ to $34 \%$ after 4 weeks. With further improvements in cell survival, PLG scaffolds may prove useful for the implantation of primary human myofibers in future clinical applications.
\end{abstract}

(C) 2007 Elsevier Ltd. All rights reserved.

Keywords: Cell viability; Muscle; Biodegradation; Tension; Poly-lactide-co-glycolide; Cell differentiation

\section{Introduction}

Skeletal muscle contains progenitor satellite cells, which are activated following injury and initiate muscle regeneration.

Abbreviations: ASGM1, anti-asialo GM1; ECM, extracellular matrix; FGF2, fibroblast growth factor 2; GFP, green fluorescent protein; HGF, hepatocyte growth factor; IgG, immunoglobulin; MGM, myoblast growth medium; NK, natural killer; NOD/SCID, non-obese diabetic/severe combined immunodeficiency; PLA, polylactide; PLG, poly-lactide-coglycolide; VEGF, vascular endothelial growth factor

* Corresponding author. Department of Pathology, Brown Medical School/The Miriam Hospital, RISE Building, 14 Third Street, Providence RI 02906, USA. Fax: + 14017934534.

E-mail address: lieven_thorrez@brown.edu (L. Thorrez).
Numerous attempts have been made to provide these cells exogenously to enhance the regeneration process and primary myoblasts can also be genetically engineered to secrete a therapeutic protein and implanted for ex vivo gene therapy [1]. Normally, transplanted proliferating myogenic cells undergo rapid and massive cell death within $48 \mathrm{~h}$ following implantation into skeletal muscle, resulting in less than $5 \%$ of transplanted cells incorporating into the host myofibers [2-4]. Proliferating myogenic cells can also be tissue engineered ex vivo into contractile 'organs' containing postmitotic myofibers (reviewed in [5]) and may prove useful in muscle regenerative and gene therapy applications [6-9]. Bioartificial muscles with postmitotic muscle fibers as a protein secretion device have a better safety profile than injecting individual 
proliferating and migrating cells because of reversibility of implants in case of an adverse event [6-9]. Ex vivo differentiated myofibers exhibit improved cell survival kinetics when compared to implanted myoblasts, with a gradual loss of viability over 30-60 days after implantation $[9,10]$ partially due to poor myofiber tension maintenance [10]. Successful transplantation of skeletal muscle cells (either myoblasts or myofibers) for structural repair or ex vivo gene therapy applications will require optimization for long-term cell survival. Recently, significant progress has been made in enhancing the survival and regenerative capacity of proliferating myogenic cells in vivo by transplanting with alginate scaffolds containing growth factors to stimulate the rapid migration of myoblasts away from the implant site $[11,12]$. Three-dimensional biodegradable polymer scaffolds have also been successfully utilized for growth, differentiation and implantation of numerous cell types, including rodent skeletal muscle cells $[13,14]$. These scaffolds are highly porous, allowing rapid diffusion of nutrients to the transplanted cells and efficient offloading of therapeutic proteins from genetically engineered cells. In addition, they can be manufactured to release angiogenic/vasculogenic factors with varying release kinetics to stimulate in-growth of host vasculature [15] which enhances transplanted cell survival [16]. To be clinically useful as cell transplant vehicles, such scaffolds need to be biocompatible without toxic side effects and clinical grade components need to be used to attach cells to the scaffold.

In the present study, we explored the use of biodegradable porous, gas-foamed poly-lactide-co-glycolide (PLG) scaffolds as an appropriate material $[15,17]$ to which primary human myoblasts could be attached using clinical grade extracellular matrix (ECM) carriers and differentiated into myofibers which could be maintained under tension. An immunodeficient non-obese diabetic/severe combined immunodeficiency (NOD/SCID) animal model was used to assess the in vivo characteristics of the constructs. We demonstrate that human myoblasts can be efficiently seeded and differentiated into postmitotic myofibers on gas-foamed PLG scaffolds and maintained under tension when implanted subcutaneously. The PLG scaffolds improve human myofiber viability in vivo relative to non-scaffold implants and therefore may be useful for future clinical applications.

\section{Materials and methods}

\subsection{Preparation of PLG scaffolds}

Salt-leached polymer scaffolds were fabricated from 85:15 PLG (Medisorb-Alkermes, Cambridge, MA) and $\mathrm{NaCl}$ particles of $250-425 \mu \mathrm{m}$, expanded using high-pressure carbon dioxide, and leached to yield pore sizes of $360 \pm 85 \mu \mathrm{m}$ as described previously $[18,19]$. PLG microspheres were prepared by standard double emulsion [20]. Scaffolds $4-13 \mathrm{~mm}$ in diameter by $1.5 \mathrm{~mm}$ thick were fabricated from particulate PLG and $10-100 \%$ (wt/wt) microsphere PLG. Scaffolds were sterilized for $30 \mathrm{~min}$ in $70 \%$ ethanol and rinsed in EBSS before cell seeding. Sterilization did not affect scaffold structure.

\subsection{Muscle progenitor cell isolation, growth and seeding}

Primary human skeletal muscle cells isolated by needle biopsy [21] were expanded in myoblast growth medium [MGM; SkGM (Cambrex Bio Science, Walkersville, MD) plus 15\% (v/v) fetal bovine serum]. Biopsies were performed on adult volunteers according to procedures approved by the Institutional Clinical Review Board of the Miriam Hospital. Cell preparations on average were $70 \%$ myogenic based on desmin-positive staining [22]. On 4.2, 7 and $13 \mathrm{~mm}$ diameter scaffolds, 1.5, 4 and 4-16 million myoblasts were seeded, respectively. Cells were first mixed with either collagen ECM $\left[0.1-0.4 \mathrm{mg} / \mathrm{ml}\right.$ Type I bovine collagen $\left(\right.$ Zyderm ${ }^{\circledR}$, Inamed Inc., Santa Barbara, CA)] or fibrin ECM $[1 \mathrm{mg} / \mathrm{ml}$ fibrinogen $+0.5 \mathrm{U} / \mathrm{ml}$ thrombin $\left(\right.$ Tisseel $^{\circledR}$, Baxter Healthcare Corp., Glendale, CA)] in a volume of 15-20, 40 and $80-100 \mu \mathrm{l}$, respectively, and seeded on top of the scaffolds. When collagen was used, scaffolds were pre-filled with collagen ECM before cell seeding. Scaffolds were incubated at $37^{\circ} \mathrm{C}$ and $5 \% \mathrm{CO}_{2}$ for $2 \mathrm{~h}$ after seeding cells, flooded with MGM and returned to the incubator for 2 days. Myoblast fusion into postmitotic myofibers was induced by incubation in differentiation medium [high-glucose DMEM (Invitrogen, Carlsbad, CA) supplemented with insulin $(10 \mu \mathrm{g} / \mathrm{ml})$, bovine serum albumin $(50 \mu \mathrm{g} / \mathrm{ml})$, epidermal growth factor $(10 \mathrm{ng} / \mathrm{ml})$ and gentamicin $(50 \mu \mathrm{g} / \mathrm{ml})]$ for an additional 3-5 days. Human myoblasts were transduced with lentiviral vectors to express green fluorescent protein (GFP), as described previously [9].

\subsection{Animal experiments}

One to four scaffolds were implanted subcutaneously into the backs of adult immunodeficient NOD.CB17-Prkdcscid/J (NOD/SCID) mice (The Jackson Laboratory, Bar Harbor, ME). All animal procedures were approved by the Harvard Institutional Animal Care and Use Committee (IACUC) and conformed to the guiding principles of the American Physiological Society. In some experiments, natural killer (NK) cells were depleted by intravenous injection of $50 \mu$ anti-asialo GM1 (ASGM1) antiserum (Wako chemicals, Dallas, TX) $2 \mathrm{~h}$ before implantation and 3, 7, 10, 14 and 21 days thereafter [23]. Control animals were injected with the same volume of normal rabbit serum (Invitrogen, Carlsbad, CA). To examine immunoglobulin (IgG) leakiness in NOD/SCID mice, IgG levels were measured by IgG Elisa (Zeptometrix, Buffalo, NY) with C57/Bl6 mice included as a positive control.

\subsection{Histology and microscopy}

Scaffold area covered with tropomyosin positive cells was quantified by counting at least nine different fields per cell number condition. GFP expression was monitored by confocal microscopy (Leica TCS SP2 AOBS). The confocal focusing location was performed on random, nonoverlapping regions of the sample. Depending on the size of the sample, this corresponded to $n=3-10$ regions, with 2-4 replicates. Confocal depth was set to 1 Airy unit. GFP area was determined with Metamorph v6.3. Where untransduced cells were used, cell viability was assessed with LIVE/ DEAD stain (Invitrogen, Carlsbad, CA). For GFP-transduced cells, viability was calculated by the average $\%$ GFP area at a specific timepoint divided by average \% GFP area on the scaffolds pre-implant. For tropomyosin staining, scaffolds were fixed in $3.7 \%$ formaldehyde, permeabilized with methanol, incubated with mouse anti-tropomyosin antibody (Sigma, St. Louis, MO) 1:100, followed by a secondary biotinylated anti-mouse $\mathrm{IgG}$ and then a preformed avidin and biotinylated horse radish peroxidase complex (Vector Laboratories, Burlingame, CA). Development was by addition of $3,3^{\prime}$-diaminobenzidene to produce a brown precipitate.

\subsection{Immunological assays}

NK cell activity was measured using a standard chromium release assay as previously described [24]. Briefly, splenocytes were harvested at 
2 or 4 weeks after scaffold implant. Target YAC-1 cells (American Type Culture Collection, Rockville, MD) were labeled with ${ }^{51} \mathrm{Cr}$ and adjusted to $5 \times 10^{4}$ cells $/ \mathrm{ml}$. Various effector to target ratios were set up in triplicate in V-bottom plates. After incubation for $4 \mathrm{~h}$ of targets and effectors at $37^{\circ} \mathrm{C}$, $100 \mu \mathrm{l}$ was removed from each well and the radioactivity present in each sample was determined using a gamma counter. The percentage-specific ${ }^{51} \mathrm{Cr}$ release was calculated using the following formula: \% specific release $=[(X-S) /(T-S)] \times 100$, where $X$ is the mean experimental release from triplicate wells, total release $(T)$ was determined from wells receiving ${ }^{51} \mathrm{Cr}$-labeled YAC-1 target cells and $1 \mathrm{~N} \mathrm{HCl}$, and spontaneous release $(S)$ was determined from wells receiving ${ }^{51} \mathrm{Cr}$-labeled YAC-1 target cells in growth medium.

\subsection{GFP protein quantification}

Total protein was extracted from the scaffolds by sonication in $1 \mathrm{ml}$ RadioImmunoPrecipitation Assay Buffer. GFP content was measured in triplicate on $200 \mu \mathrm{l}$ samples by fluorimetric analysis (excitation $485 \mathrm{~nm}-$ emission 528) on a Synergy HT microplate reader (Biotek Winooski, VT).

\subsection{Statistics}

Data are presented as mean \pm standard error of the mean and statistical analyses were performed in Microsoft Excel for Mac using an unpaired $t$-test. Bonferroni correction was applied when testing multiple groups.

\section{Results}

\subsection{Differentiation of human muscle cells on PLG scaffolds in vitro}

Cells were seeded at various densities (4-16 million) on $13 \mathrm{~mm}$ scaffolds, composed of particulate PLG and $10 \%$ (wt/wt) microsphere PLG. Human primary myoblasts were first mixed with an ECM solution and applied to the scaffolds; the ECM was required for retention of a majority of the cells on the PLG scaffold (data not shown). Two clinical grade products were utilized, either collagen type I $\left(\right.$ Zyderm ${ }^{\circledR}$ ) or fibrin $\left(\right.$ Tisseel ${ }^{\circledR}$ ) as the cell carrier. The pore size of $360 \pm 85 \mu \mathrm{m}$ allowed for a quick penetration of the cell-ECM mix in the interconnected scaffold pores. When collagen I was used as the ECM, it took $10-30 \mathrm{~min}$ to solidify at $37^{\circ} \mathrm{C}$, allowing the cell-ECM mix to penetrate throughout the scaffold and leak out. To prevent this in subsequent experiments, scaffolds were prefilled with cellfree collagen and allowed to solidify before adding the cell-ECM mix to the top of the scaffolds. When fibrin was used as the ECM, prefilling was not necessary due to a much faster clotting time $(<90 \mathrm{~s})$. In both cases, the cell-ECM mix formed a high-density layer of myoblasts on top of the scaffolds. When scaffolds were incubated in differentiation medium for 4 days, a dense layer of postmitotic muscle fibers expressing sarcomeric tropomyosin was observed primarily on top of the scaffold (Fig. 1), indicating efficient differentiation into myofibers. Scaffold surface myofiber density was dependent on initial cell seeding number. For scaffolds plated with 4 million cells, $54 \pm 9 \%$ of the scaffold area was covered with tropomyosin positive differentiated muscle fibers by days $8-10$ postplating while $72 \pm 9 \%$ was tropomyosin positive when 16 million cells were used for the initial plating. Cell densities up to 16 million/scaffold did not influence their differentiation potential (Fig. 1). As expected, myofibers were not aligned in any specific direction. Fiber density decreased from the surface of the scaffolds to the middle (data not shown). Plating the 4-8 million cells with either ECMs with these techniques resulted in the formation of an approximately $100 \mu \mathrm{m}$ thick cell layer of well formed postmitotic muscle fibers on the surface of the scaffolds (Fig. 1). With these protocols, myofibers were retained under tension in vitro on top of the gas-foamed PLG scaffolds for at least several weeks. We attempted to seed syngeneic murine GFP-transduced myoblasts on PLG scaffolds in a collagen:Matrige ${ }^{\circledR}$, collagen or fibrin ECM. In contrast to human myoblasts, the murine myofibers consistently rolled up into clumps and detached from the surface of the scaffold within 2-4 days of differentiation (data not shown). Scaffolds were also made containing $25 \%, 50 \%$ or $100 \%$ PLG microspheres and seeded with 16 million GFP expressing myoblasts. Differentiation was not affected by either varying the microsphere content of the scaffolds, or the composition of the carrier ECM. After 7-8 days in vitro, GFP myofiber retention was studied by confocal microscopy. Inclusion of a higher percentage microsphere PLG in the scaffolds improved the density of differentiated myofibers on the scaffold (Fig. 2). For subsequent experiments, PLG scaffolds containing $50-100 \%$ microspheres were used with either fibrin or collagen ECM.

\subsection{Muscle fiber survival on the scaffolds in vivo}

Non-transduced or GFP-transduced myoblasts were differentiated into myofibers on $13 \mathrm{~mm}$ scaffolds containing 100\% PLG microspheres and implanted subcutaneously in the dorsal region of immunodeficient NOD/ SCID mice for up to 1 month. For non-GFP cells, living cells were stained green with membrane permeable calcein AM and dead cells were stained red by membrane impermeant ethidium homodimer-1. Cell survival at various depths was determined by confocal microscopy. At the surface of the scaffold, many GFP positive cells could be observed (Fig. 3A). Longitudinal myofiber morphology was retained on the scaffolds even after 30 days, indicating that the fibers could be kept under tension on the scaffolds (Fig. 3A). At 50-70 $\mu \mathrm{m}$ depth, many dead cells were observed (Fig. 3B) and at $150 \mu \mathrm{m}$ depth exclusively dead cells were seen (Fig. 3C).

Primary human skeletal myoblasts stably transduced with GFP were used to track in vivo myofiber survival by confocal microscopy. Myoblasts were embedded in a fibrin or zyderm ECM and seeded on $4.2 \mathrm{~mm}$ scaffolds, composed of either $50 \%$ or $100 \%$ microspheres. Scaffolds were implanted subcutaneously in the dorsal region of NOD/SCID mice and explanted for analysis at 2 and 4 weeks in vivo. Scaffolds were imaged by confocal microscopy (3-10 fields/scaffold, 4 scaffolds/group) 

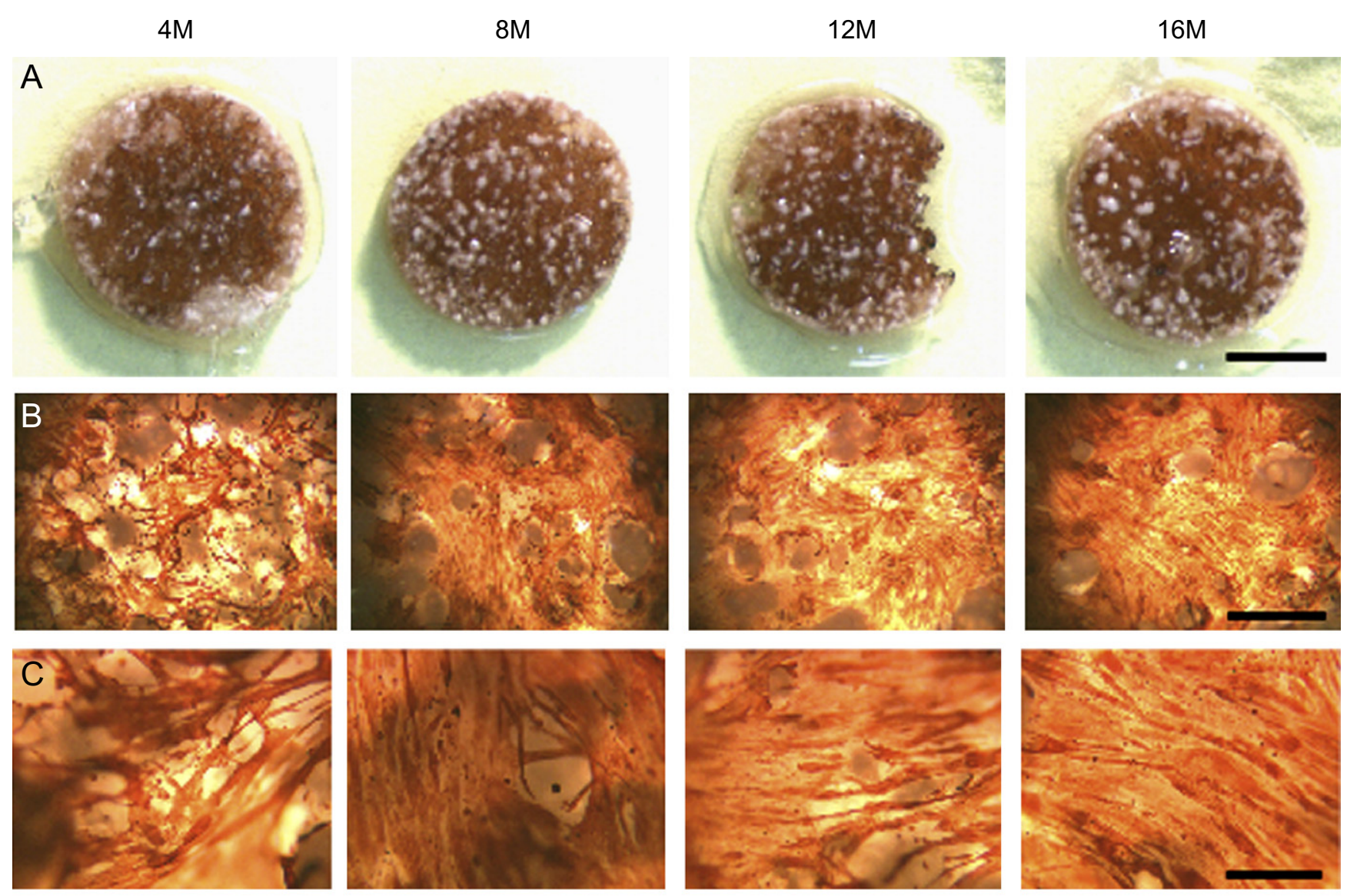

D

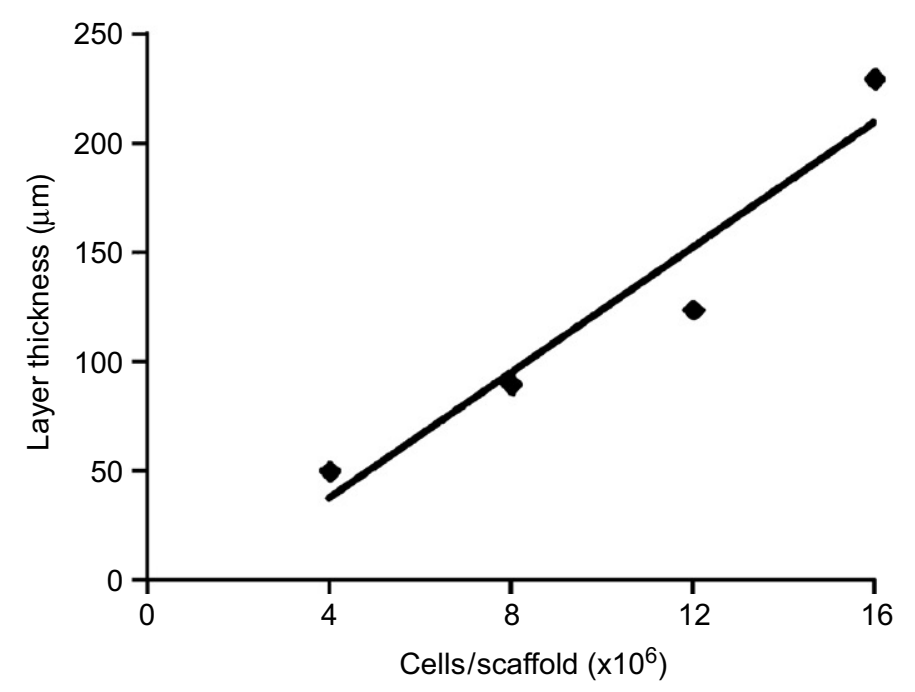

Fig. 1. Differentiation of human skeletal myoblasts to myofibers on PLG scaffolds: various numbers $(4,8,12$ or 16 million cells, indicated on top of figure) of human myoblasts were seeded in $0.1 \mathrm{mg} / \mathrm{ml}$ collagen ECM and differentiated to myofibers on $13 \mathrm{~mm}$ PLG scaffolds containing $10 \%$ microspheres and prefilled with $0.1 \mathrm{mg} / \mathrm{ml}$ collagen ECM. After 5 days in differentiation medium, cells were stained for the expression of sarcomeric tropomyosin (brown color). Myofibers were short and not aligned in any specific direction. (A) Picture of entire scaffold. Scale bar represents $5 \mathrm{~mm}$. (B) $2.5 \times$ objective, scale bar represents $1 \mathrm{~mm}$. (C) $10 \times$ objective, scale bar represents $0.25 \mathrm{~mm}$. (D) Cell layer thickness was measured on thin sections of paraffin embedded scaffolds and is linearly dependent on the initial number of seeded cells.

pre-implant and at 2 or 4 weeks in vivo and GFP area was quantified with Metamorph software. Results are summarized in Fig. 4. To calculate cell survival at a specific timepoint, GFP area at that time point was normalized to
GFP area pre-implant. At 2 weeks in vivo, myofibers embedded in a zyderm ECM survived better than myofibers in the fibrin ECM (viability $77 \pm 8 \%$ versus $52 \pm 8 \%$ ); however, this difference was not statistically 

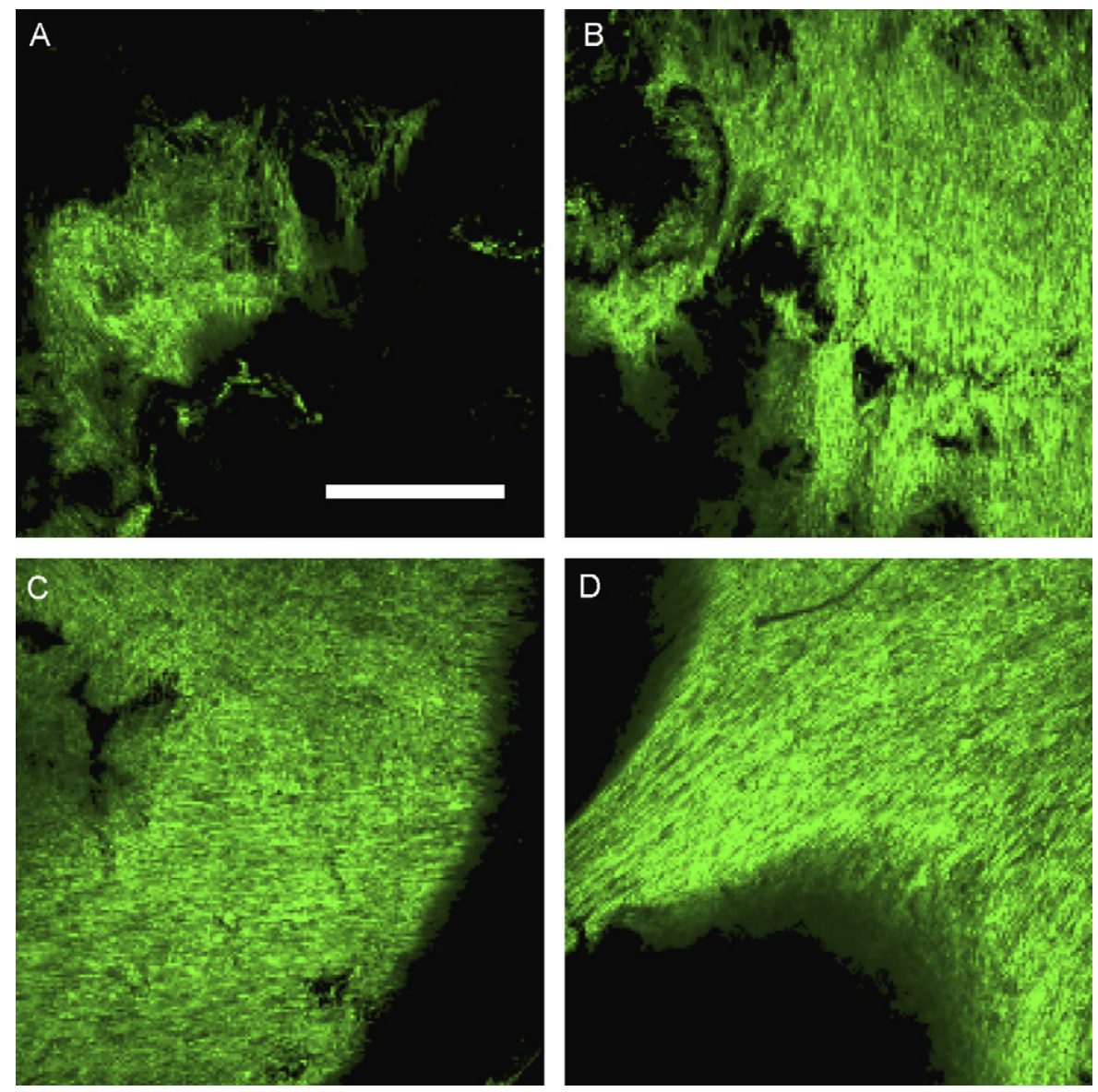

Fig. 2. Growth of GFP human skeletal muscle cells on PLG scaffolds with varying microsphere content: sixteen million myoblasts in fibrin ECM were plated as described in Materials and methods on the $13 \mathrm{~mm}$ diameter scaffolds containing $10 \%, 25 \%, 50 \%$ or $100 \%$ (w/w) microspheres. After $7-8$ days in vitro, cells were visualized by confocal microscopy. Images are composites of ten $1.7 \mu \mathrm{m}$ sections of the top layer of the scaffolds: (A) $10 \%$; (B) $25 \%$; (C) $50 \%$; and (D) $100 \%$. Scale bar represents $500 \mu \mathrm{m}$.
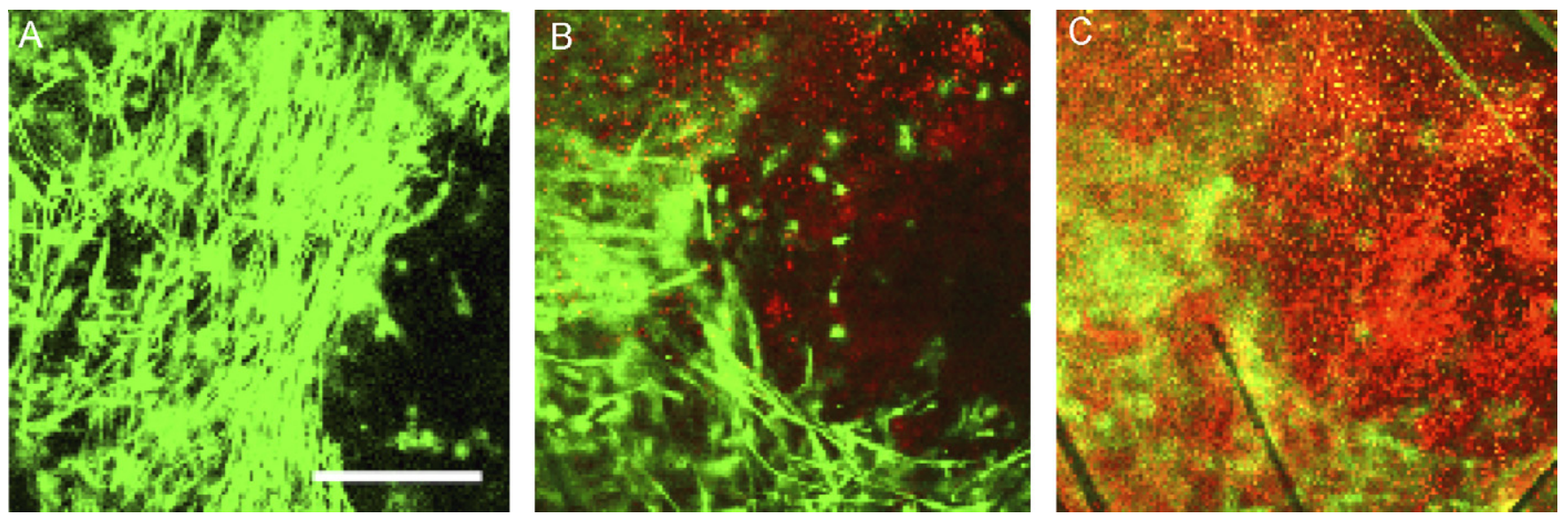

Fig. 3. Myoblast cell survival in vivo at various depths in the scaffold: myoblasts were seeded on the PLG scaffolds in a fibrin ECM, differentiated to myofibers and implanted subcutaneously in the back of NOD/SCID mice for 1 month. Living cells were stained with membrane permeable calcein AM (green) and dead cells were stained red by membrane impermeant ethidium homodimer-1. Cell survival at various depths was imaged by confocal microscopy: (A) surface of scaffold, (B) 50-70 $\mu \mathrm{m}$ depth and (C) $150 \mu \mathrm{m}$ depth. Scale bar represents $250 \mu \mathrm{m}$.

significant ( $t$-test with Bonferroni correction). There was no significant difference in cell survival between the $50 \%$ and $100 \%$ microspheres scaffold compositions. At 4 weeks, cell survival for the different conditions was similar for both collagen and fibrin ECM. Average survival in scaffolds at 4 weeks with fibrin ECM was $24 \pm 3 \%$, and $21 \pm 4 \%$ in the collagen ECM. Overall survival of the muscle cells was $66 \pm 6 \%$ at 2 weeks and $22 \pm 2 \%$ at 4 weeks. 


\subsection{Degradation of $P L G$}

Beginning 2 weeks after scaffold implantation in vivo, a transparent material was observed covering or attached to parts of the PLG scaffolds. This material was observed independently of microsphere content or use of fibrin or collagen ECM and increased with time in vivo. Since the material was also observed on scaffolds not containing cells (Fig. 5A), it cannot be attributed to the implanted myofibers. The material appeared to have a crystalline structure by light microscopy (Fig. 5B and C). Interestingly, the size of these microscopic structures was similar to the size of the $\mathrm{NaCl}$ crystals which were used to create the pores of the scaffolds $(250-425 \mu \mathrm{m})$. The clear film did not dissolve in chloroform, a solvent for PLG, and was also resistant to collagenase treatment. Its impact on muscle cell survival is unknown.

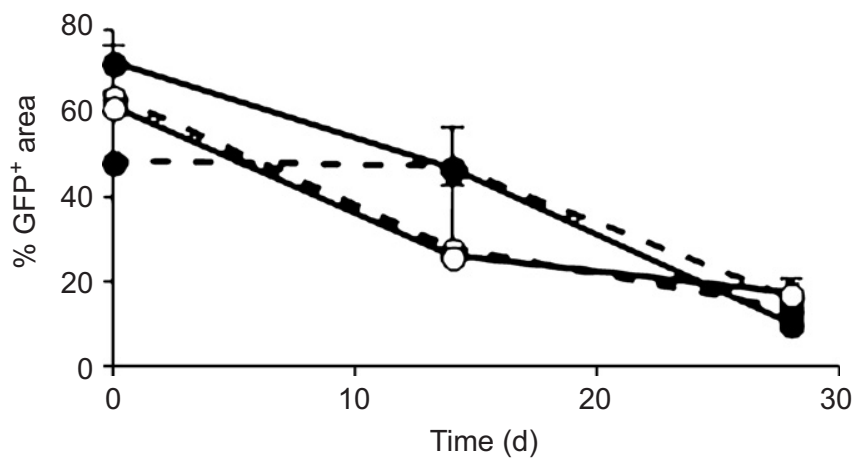

Fig. 4. Cell survival as a function of ECM and scaffold composition: scaffolds ( $4.2 \mathrm{~mm}$ diameter) composed of either $100 \%$ microspheres (solid lines) or $50 \%$ microspheres (dotted lines) and seeded with 1.5 million human GFP human myoblasts were implanted subcutaneously in the back of NOD/SCID mice. Cells were embedded in a fibrin ECM (open circles) or collagen ECM (filled circles). Datapoints represent GFP area as quantified by confocal analysis pre-implant and after 2 or 4 weeks in vivo. At 2 weeks in vivo, myofibers embedded in a collagen ECM on average covered more area than myofibers in the fibrin ECM; however, this difference was not statistically significant. At 4 weeks, GFP area for all conditions was $10-17 \%(22 \pm 2 \%$ survival when area was normalized to preimplant GFP area), with no statistical differences for the tested conditions.

\subsection{Effect of NK cell depletion}

NOD/SCID mice are deficient in T and B cell function, complement deficient and have low levels of NK cells [25]. We examined whether these residual NK cells contributed to the decreased cell survival in vivo. All mice were initially tested for leakiness by immunoglobulin G enzyme linked immunosorbent assay and found to have IgG levels $<0.5 \mu \mathrm{g} / \mathrm{ml}$ plasma, whereas C57/B16 controls had IgG levels $>5000 \mu \mathrm{g} / \mathrm{ml}$ (data not shown). Seven mm diameter PLG scaffolds containing 100\% microspheres were seeded with 4 million GFP myoblasts in a fibrin ECM and after differentiation implanted subcutaneously in NOD/ SCID animals. Half of the animals received intravenous (iv) infusions of anti-ASGM1 antibody, effectively depleting the residual NK cells as determined by a cytotoxicity test (data not shown), whereas the control group received isotype serum injections. GFP area on the scaffolds was quantified by confocal microscopy pre-implant and after 2 or 4 weeks in vivo (Fig. 6A). No significant difference in myofiber survival was apparent at week 2 (NK depleted: $57 \pm 5 \%$; control: $55 \pm 3 \%$ ), but at 4 weeks, myofiber survival in the NK depleted group was significantly higher than control: $34 \pm 2 \%$ in the NK depleted group versus $22 \pm 2 \%$ in the control group $(p<0.05)$.

To confirm these results, total protein was extracted from the scaffolds at week 4 and GFP fluorescence from the extracts was assessed by fluorimetric analysis. Data were normalized to the fluorescence from scaffolds in the control group. Relative GFP content of the scaffolds in the NK depleted animals was two-fold higher than controls $(p<0.05$, Fig. 6B).

\section{Discussion}

In this study, we investigated the potential of biodegradable PLG gas-foamed scaffolds as structures for skeletal myoblast seeding, differentiation and in vivo myofiber survival. Since PLG with high lactic acid content (85:15) exhibits superior mechanical properties [26], this copolymer was chosen to ensure the scaffold would remain intact
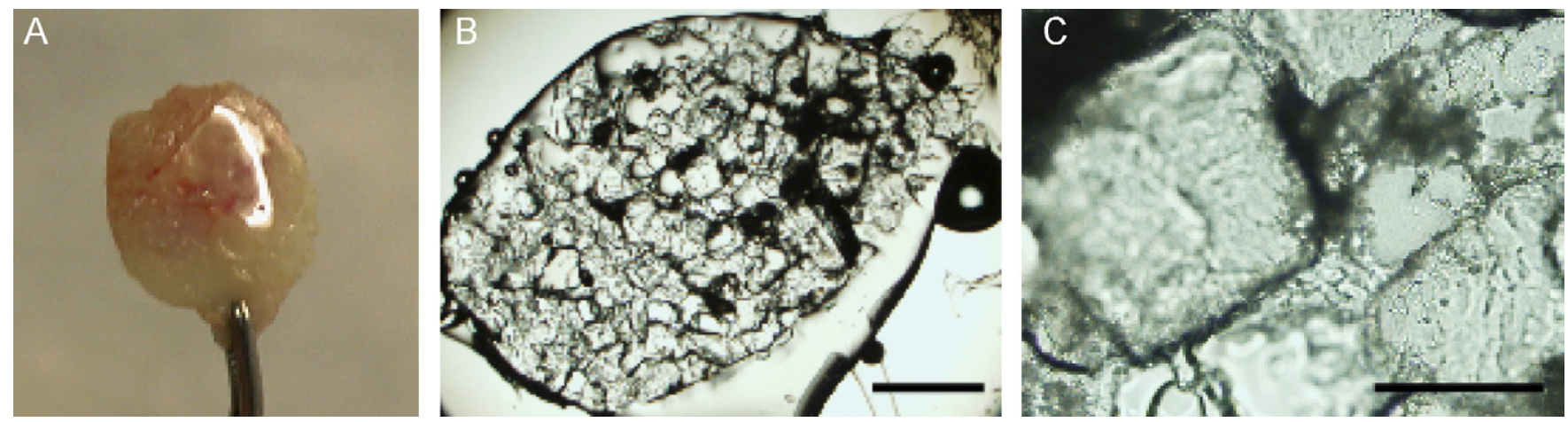

Fig. 5. Degradation products: (A) PLG scaffold prefilled with $0.4 \mathrm{mg} / \mathrm{ml}$ collagen ECM, not containing cells was implanted subcutaneously in a NOD/ SCID mouse for 47 days. A transparent product covers part of the scaffold. (B) Brightfield microscopic examination revealed a crystalline structure. Scale bar represents $1 \mathrm{~mm}$. (C) Detail of the crystalline structure in the transparent film. Scale bar represent $250 \mu \mathrm{m}$. 
A

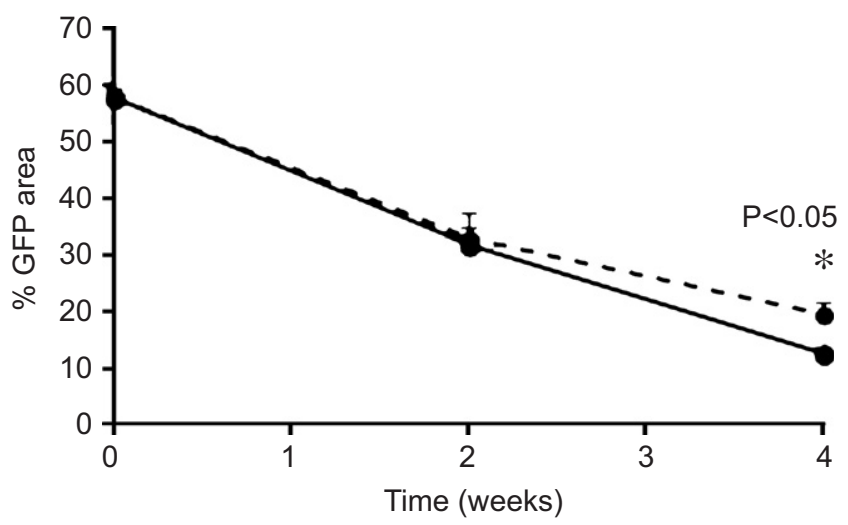

B

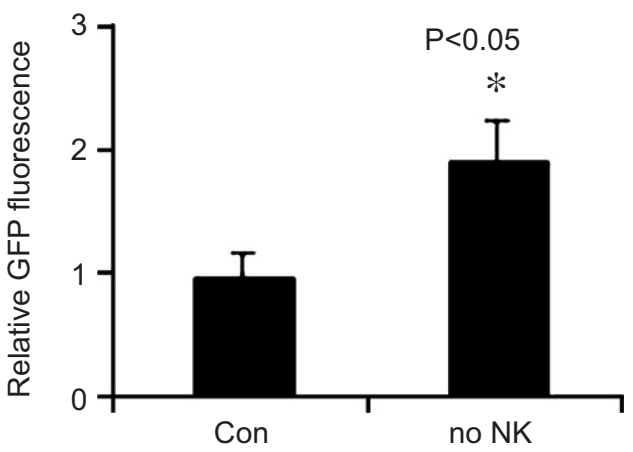

Fig. 6. Effect of NK cell depletion on cell viability: $100 \%$ microsphere, $7 \mathrm{~mm}$ diameter PLG scaffolds were seeded with 4 million GFP myoblasts in a fibrin ECM and implanted subcutaneously in NOD/SCID animals. (A) GFP area on the scaffolds was quantified by confocal microscopy preimplant and after 2 or 4 weeks in vivo. In the NK cell depleted group (dotted line), animals received intravenous injections of anti-ASGM1 antibody, while control group (solid line) received an isotype serum control. No significant difference was apparent at week 2, but at week 4 , myofiber survival in the NK depleted group was significantly $(p<0.05)$ higher than control. (B) Scaffolds in the NK depleted animals contained two-fold as much GFP protein compared to the control group $(p<0.05)$ after 4 weeks in vivo.

for the duration of the in vivo studies. The matrix of the scaffolds was formed by foaming PLG with $\mathrm{NaCl}$ in $\mathrm{CO}_{2}$ to obtain a highly porous $(94.6 \pm 0.9 \%)$ structure with largely open, interconnected pores [18,26,27]. Pore size of the PLG scaffolds was $360 \pm 85 \mu \mathrm{m}$ [18], which allows for an even distribution of seeded cells, infiltration by host cells and vascularization [28]. These scaffolds were described to be suitable for cell adherence, proliferation and formation of new three-dimensional tissues $[16,18]$.

For gene therapy applications, skeletal muscle myofibers have a very strong protein production capacity, are nondividing and non-migratory and thus have a favorable safety perspective compared to proliferating migratory myogenic cells since the attached cells would be retrievable if an adverse event occurs [6,10,22]. For clinical use, the components used for implantation need to be FDA approved. PLG has been used in the clinic for more than
3 decades in biodegradable sutures and has more recently also been used for fracture fixation devices and drug delivery systems. It has good biocompatibility and implantation profiles in bone or soft tissues, causing none or only a mild inflammatory response which diminishes with time [29]. To retain cells in or on scaffolds an ECM carrier such as Matrigel ${ }^{\mathrm{TM}}$ is often being used. Tisseel ${ }^{\circledR}$ and Zyderm ${ }^{\circledR}$ are two FDA approved ECM products used commonly as a tissue glue and bulking agent, respectively, and were used in this study.

Human myoblasts could be efficiently seeded on PLG scaffolds, and both the fibrin or collagen ECM could be used to retain the cells on the scaffold. Myoblasts could subsequently be differentiated into postmitotic myofibers. When fibrin was employed as an ECM, myoblasts did not penetrate deeply into the scaffold pores due to the rapid solidification of the fibrin ECM (less than $90 \mathrm{~s}$ ). In contrast, the gelling time of collagen ECM was significantly longer (10-30 min) and resulted in a penetration of cells deeper into the pores of the scaffolds, and seeping out of the cell-ECM mixture. Subsequently, scaffolds were prefilled with collagen ECM to limit cell-ECM penetration into the scaffolds and cell-ECM loss during the seeding process. Using these procedures, myofibers formed upon incubation in a differentiation medium, and were randomly oriented in a well-defined layer on top of the scaffold, with good myofiber retention in vitro for at least 4 weeks. The gasfoamed PLG scaffold thus appears to be an adequate method for transferring differentiated myofibers under tension for in vivo studies.

Following implantation of the PLG scaffolds subcutaneously in the NOD-SCID mice for several weeks, we observed a clear plastic-like product around the implants, which was seen when either collagen or fibrin ECM was used. To our knowledge, such material has not been reported before. Generation of this substance, which consists of small adjacent crystals, is not attributable to myofibers, since it also appeared when scaffolds were implanted without cells. PLG undergoes hydrolysis to produce the original monomers, lactic acid and glycolic acid, which are by-products of various metabolic pathways in the body. Polymer impurity is a possible explanation for the occurrence of this PLG degradation product. A much lower incidence of late-stage foreign body reactions has been observed with purified melt processed polylactide (PLA) than with bulk PLA, which may have contained a relatively high level of residual lactide [30]. Partial recrystallization of lactide monomers may have led to formation of this material on the surface of the implants. Poly-l-lactic acid polymer has a crystallinity which is in agreement with our observations. It has also been suggested that the occurrence of inflammatory reactions may depend on the anatomical region, the capacity of the tissue to clear the degradation products, and the volume of polymer implanted [31]. The contribution of this material to myofiber loss from the scaffold with time in vivo as seen in the present study is unknown. 
In a previous study, human myofibers were embedded only in a collagen ECM and implanted subcutaneously in NOD/SCID mice without tension [9]. Cell viability decreased $91 \pm 4 \%$ over 4 weeks, and could not be improved by delivery of vascular endothelial growth factor $(\mathrm{VEGF})_{165}$ to stimulate vascularization of the implant site [9]. It was hypothesized that loss of cell viability may be partly explained by the lack of tension on the myofibers. Since human myofibers could be kept under tension on PLG scaffolds in vivo we were able to study the effect of tensioning on cell survival. Overall cell survival of the tensioned myofibers was $66 \pm 6 \%$ at 2 weeks and $22 \pm 2 \%$ at 4 weeks, which is two-fold higher than untensioned myofibers as reported previously [9]. Using either fibrin or collagen type I as the ECM, or varying the percentage of PLG microspheres did not affect this cell viability.

NOD/SCID mice have become a standard model for in vivo evaluation of human hematopoietic stem cell function [32]. They are not only deficient in T and B cells, but also exhibit macrophage dysfunction and an absence of circulating complement [25]. NOD/SCID mice are often referred to as immunodeficient, yet there is still a low level of NK cells present and active, which may be involved in rejection of xenogenic cell transplants [33-36]. Depletion of NK activity by antibody treatment [36] or use of $\beta 2$-microglobulin-deficient NOD/SCID mice [35] was demonstrated to improve engraftment of human cells. Antibody depletion of NK cells led to $50 \%$ higher muscle cell survival at 4 weeks after implantation. Even with NK depletion, nearly $70 \%$ of muscle cells died by 4 weeks in vivo. Thus, other components of the innate immune system may still affect the survival of the xenogeneic human cells. When human mesenchymal stem cells were implanted on poly ( $\varepsilon$-caprolactone) in a NOD/SCID model, host macrophages along with neutrophils and mast cells slowly invaded the implants and the majority of the implanted cells were eliminated by 2 weeks [33]. We thus cannot exclude that cell loss is partly due to other innate immune functions, such as macrophage activity. Macrophages have been detected in the spleens of NOD/SCID mice, however, they are incapable of secreting IL-1 in response to stimulation with lipopolysaccharides and therefore are thought to be functionally immature [25,37]. Use of other immunodeficient animal models may enhance xenogeneic cell survival. Gamma c ${ }^{-} / \mathrm{RAG}^{-}$[38] and NOD/SCID/gamma c $\mathrm{c}^{-}$mice [39] are completely T, B and NK cell negative and have been found to improve survival of implanted human myoblasts compared to SCID mice [40]. Nevertheless it was observed that human hematopoietic stem cells still needed exogenous cytokine administration in these animals for an enhanced engraftment [41].

An alternative hypothesis for poor cell survival in the xenogeneic setting may be a lack of one or more local growth/survival factors. Murine (growth factor) proteins may not be efficiently recognized by human cell receptors and therefore may contribute to impaired growth/survival of the xenogeneic cells. The use of syngeneic (or autologous for use in human patients) cells is warranted for optimal cell survival and avoiding immune responses. Indeed, implanting syngeneic myofibers in the NOD/SCID animal model resulted in much better cell survival, even in the absence of tension [9]. Delivery of recombinant growth factors at defined times after cell implantation may be beneficial to further increase cell viability and protein secretion capacity. Anabolic growth factors such as insulinlike growth factor $1[9,42]$ or growth hormone [6] can stimulate myofiber hypertrophy or attenuate atrophy. The use of angiogenic molecules such as VEGF may be beneficial to rapidly induce a functional blood vessel supply to the transplanted myofibers [8,9]. Delivery of multiple growth factors can be precisely controlled by incorporation of PLG microspheres containing growth factors in the scaffolds $[15,26,43]$. Hepatocyte growth factor (HGF) and fibroblast growth factor 2 (FGF2), which have been used to stimulate myoblast migration in other studies [12] may not be optimal for the postmitotic muscle fibers. In a therapeutic approach where genetically modified myofibers attached to a scaffold are used for longterm protein secretion, cell migration would be an undesirable feature since retrievability of genetically engineered tissue in case of an adverse response is one of the key advantages of using non-migratory myofibers.

Survival of myofibers on the PLG scaffolds compares favorably to use of other biomaterials for in vivo muscle cell survival. Alginate hydrogels containing cell adhesion peptides had a murine myoblast survival of $16 \%$ over a $24 \mathrm{~h}$ time period [11]. Optimal scaffold design with sustained delivery of HGF and FGF2 increased viability to $40 \%$ at $96 \mathrm{~h}$. Cell viability on PLG scaffolds as described in this paper compares favorably with the alginate scaffolds, even without growth factor integration.

\section{Conclusions}

Biodegradable polymer scaffolds based on PLG provide a reliable method for the successful transfer of ex vivo differentiated postmitotic human muscle fibers in vivo. Using these scaffolds, myofibers remain under tension, doubling their long-term viability. Therefore, these PLG scaffolds may be important for future use in human muscle repair or gene therapy applications.

\section{Acknowledgments}

The authors would like to thank Claudia Fishbach and Will Yuen for help with the scaffold preparation and Gilbert DiLeone for assistance with the NK assay. This research was funded through a Fellowship from the Belgian American Educational Foundation (LT), a NIH Ruth L. Kirschstein Senior Fellowship (HV), The Miriam Hospital, EUFP6 INTHER LSHB-CT-2005018961 and Fund for Scientific Research, Flanders (FWO). The authors confirm that there are no known conflicts of interest associated with this publication and there has been 
no significant financial support for this work that could have influenced its outcome.

\section{References}

[1] Blau HM, Springer ML. Muscle-mediated gene-therapy. N Engl J Med 1995;333(23):1554-6.

[2] Skuk D, Caron NJ, Goulet M, Roy B, Tremblay JP. Resetting the problem of cell death following muscle-derived cell transplantation: detection, dynamics and mechanisms. J Neuropathol Exp Neurol 2003;62(9):951-67.

[3] Beauchamp JR, Morgan JE, Pagel CN, Partridge TA. Dynamics of myoblast transplantation reveal a discrete minority of precursors with stem cell-like properties as the myogenic source. J Cell Biol 1999; 144(6):1113-22.

[4] Qu Z, Balkir L, van Deutekom JC, Robbins PD, Pruchnic R, Huard J. Development of approaches to improve cell survival in myoblast transfer therapy. J Cell Biol 1998;142(5):1257-67.

[5] Kosnik P, Dennis JE, Vandenburgh H. Tissue engineering skeletal muscle. In: Guilah F, editor. Functional tissue engineering. New York: Springer; 2003. p. 392.

[6] Vandenburgh H, Del Tatto M, Shansky J, Goldstein L, Russell K, Genes N, et al. Attenuation of skeletal muscle wasting with recombinant human growth hormone secreted from a tissueengineered bioartificial muscle. Hum Gene Ther 1998;9(17):2555-64.

[7] Lu Y, Shansky J, Del Tatto M, Ferland P, McGuire S, Marszalkowski J, et al. Therapeutic potential of implanted tissue-engineered bioartificial muscles delivering recombinant proteins to the sheep heart. Ann N Y Acad Sci 2002;961:78-82.

[8] Lu Y, Shansky J, Del Tatto M, Ferland P, Wang X, Vandenburgh H. Recombinant vascular endothelial growth factor secreted from tissueengineered bioartificial muscles promotes localized angiogenesis. Circulation 2001;104(5):594-9.

[9] Thorrez L, Vandenburgh H, Callewaert N, Mertens N, Shansky J, Wang L, et al. Angiogenesis enhances factor ix delivery and persistence from retrievable human bioengineered muscle implants. Mol Ther 2006; 14(3):442-51.

[10] Vandenburgh H, Del Tatto M, Shansky J, Lemaire J, Chang A, Payumo F, et al. Tissue-engineered skeletal muscle organoids for reversible gene therapy. Hum Gene Ther 1996;7(17):2195-200.

[11] Hill E, Boontheekul T, Mooney DJ. Designing scaffolds to enhance transplanted myoblast survival and migration. Tissue Eng 2006;12(5): 1295-304.

[12] Hill E, Boontheekul T, Mooney DJ. Regulating activation of transplanted cells controls tissue regeneration. Proc Natl Acad Sci USA 2006;103(8):2494-9.

[13] Riboldi SA, Sampaolesi M, Neuenschwander P, Cossu G, Mantero S. Electrospun degradable polyesterurethane membranes: potential scaffolds for skeletal muscle tissue engineering. Biomaterials 2005;26(22):4606-15.

[14] Levenberg S, Rouwkema J, Macdonald M, Garfein ES, Kohane DS, Darland DC, et al. Engineering vascularized skeletal muscle tissue. Nat Biotechnol 2005;23(7):879-84.

[15] Richardson TP, Peters MC, Ennett AB, Mooney DJ. Polymeric system for dual growth factor delivery. Nat Biotechnol 2001;19(11): 1029-34.

[16] Smith MK, Peters MC, Richardson TP, Garbern JC, Mooney DJ. Locally enhanced angiogenesis promotes transplanted cell survival. Tissue Eng 2004;10(1-2):63-71.

[17] Lee KY, Peters MC, Anderson KW, Mooney DJ. Controlled growth factor release from synthetic extracellular matrices. Nature 2000; 408(6815):998-1000.

[18] Harris LD, Kim BS, Mooney DJ. Open pore biodegradable matrices formed with gas foaming. J Biomed Mater Res 1998;42(3):396-402.

[19] Mooney DJ, Baldwin DF, Suh NP, Vacanti JP, Langer R. Novel approach to fabricate porous sponges of poly(D,L-lactic- $c o$-glycolic acid) without the use of organic solvents. Biomaterials 1996;17(14): 1417-22.

[20] Cohen S, Yoshioka T, Lucarelli M, Hwang LH, Langer R. Controlled delivery systems for proteins based on poly(lactic/glycolic acid) microspheres. Pharm Res 1991;8(6):713-20.

[21] Hennessey JV, Chromiak JA, Della Ventura S, Guertin J, MacLean DB. Increase in percutaneous muscle biopsy yield with a suction-enhancement technique. J Appl Physiol 1997;82(6):1739-42.

[22] Powell C, Shansky J, Del Tatto M, Forman DE, Hennessey J, Sullivan $\mathrm{K}$, et al. Tissue-engineered human bioartificial muscles expressing a foreign recombinant protein for gene therapy. Hum Gene Ther 1999;10(4):565-77.

[23] Yamanokuchi S, Ikai I, Nishitai R, Matsushita T, Sugimoto S, Shiotani T, et al. Asialo GM1 positive CD8 $+\mathrm{T}$ cells induce skin allograft rejection in the absence of the secondary lymphoid organs. J Surg Res 2005;129(1):57-63.

[24] Fast LD. Identification of a single non- $\mathrm{H}-2$ gene regulating graftversus-host disease response. J Immunol 1990;144(11):4177-82.

[25] Shultz LD, Schweitzer PA, Christianson SW, Gott B, Schweitzer IB, Tennent B, et al. Multiple defects in innate and adaptive immunologic function in NOD/LtSz-scid mice. J Immunol 1995;154(1):180-91.

[26] Sheridan MH, Shea LD, Peters MC, Mooney DJ. Bioabsorbable polymer scaffolds for tissue engineering capable of sustained growth factor delivery. J Control Release 2000;64(1-3):91-102.

[27] Mooney DJ, Sano K, Kaufmann PM, Majahod K, Schloo B, Vacanti $\mathrm{JP}$, et al. Long-term engraftment of hepatocytes transplanted on biodegradable polymer sponges. J Biomed Mater Res 1997;37(3): 413-20.

[28] Peters MC, Polverini PJ, Mooney DJ. Engineering vascular networks in porous polymer matrices. J Biomed Mater Res 2002;60(4):668-78.

[29] Avgoustakis K. Polylactic-co-glycolic acid (PLGA). In: Bowlin GL, editor. Encyclopedia of biomaterials and biomedical engineering. Dekker, 2005.

[30] Perrin DE, English JP. Polyglycolide and polylactide. In: Domb AJ, Kost J, Wiseman DM, editors. Handbook of biodegradable polymers. Amsterdam: Harwood Academic Publishers; 1997. p. 3-27.

[31] Athanasiou KA, Niederauer GG, Agrawal CM. Sterilization, toxicity, biocompatibility and clinical applications of polylactic acid/polyglycolic acid copolymers. Biomaterials 1996;17(2):93-102.

[32] Greiner DL, Hesselton RA, Shultz LD. SCID mouse models of human stem cell engraftment. Stem Cells 1998;16(3):166-77.

[33] Xia Z, Ye H, Choong C, Ferguson DJ, Platt N, Cui Z, et al. Macrophagic response to human mesenchymal stem cell and poly(epsilon-caprolactone) implantation in nonobese diabetic/severe combined immunodeficient mice. J Biomed Mater Res A 2004;71(3): $538-48$.

[34] Lin Y, Chen Y, Zeng Y, Wang T, Zeng S. Lymphocyte phenotyping and NK cell activity analysis in pregnant NOD/SCID mice. J Reprod Immunol 2005;68(1-2):39-51.

[35] Christianson SW, Greiner DL, Hesselton RA, Leif JH, Wagar EJ, Schweitzer IB, et al. Enhanced human CD4 $+\mathrm{T}$ cell engraftment in beta2-microglobulin-deficient NOD-scid mice. J Immunol 1997; 158(8):3578-86.

[36] Yoshino H, Ueda T, Kawahata M, Kobayashi K, Ebihara Y, Manabe A, et al. Natural killer cell depletion by anti-asialo GM1 antiserum treatment enhances human hematopoietic stem cell engraftment in NOD/Shi-scid mice. Bone Marrow Transplant 2000;26(11):1211-6.

[37] Serreze DV, Gaedeke JW, Leiter EH. Hematopoietic stem-cell defects underlying abnormal macrophage development and maturation in NOD/Lt mice: defective regulation of cytokine receptors and protein kinase C. Proc Natl Acad Sci USA 1993;90(20):9625-9.

[38] Goldman JP, Blundell MP, Lopes L, Kinnon C, Di Santo JP, Thrasher AJ. Enhanced human cell engraftment in mice deficient in RAG2 and the common cytokine receptor gamma chain. Br J Haematol 1998;103(2):335-42.

[39] Ito M, Hiramatsu H, Kobayashi K, Suzue K, Kawahata M, Hioki K, et al. NOD/SCID/gamma(c)(null) mouse: an excellent recipient 
mouse model for engraftment of human cells. Blood 2002;100(9): 3175-82.

[40] Cooper RN, Irintchev A, Di Santo JP, Zweyer M, Morgan JE, Partridge TA, et al. A new immunodeficient mouse model for human myoblast transplantation. Hum Gene Ther 2001;12(7):823-31.

[41] Mazurier F, Fontanellas A, Salesse S, Taine L, Landriau S, Moreau-Gaudry F, et al. A novel immunodeficient mouse model-RAG $2 \times$ common cytokine receptor gamma chain double mutants - requiring exogenous cytokine administration for human hematopoietic stem cell engraftment. J Interferon Cytokine Res 1999; 19(5):533-41.

[42] Shansky J, Creswick B, Lee P, Wang X, Vandenburgh H. Paracrine release of insulin-like growth factor 1 from a bioengineered tissue stimulates skeletal muscle growth in vitro. Tissue Eng 2006;12(7): 1833-41.

[43] Ennett AB, Kaigler D, Mooney DJ. Temporally regulated delivery of VEGF in vitro and in vivo. J Biomed Mater Res A 2006;79(1): 176-84. 